\title{
Pyogenic liver abscess
}

\author{
Gwilym James Webb, ${ }^{1}$ Thomas Patrick Chapman, ${ }^{1}$ Philip John Cadman, ${ }^{2}$ \\ David Angelo Gorard ${ }^{1}$
}

\begin{abstract}
${ }^{1}$ Department of
Gastroenterology, Wycombe Hospital, High Wycombe,

Buckinghamshire, UK

${ }^{2}$ Department of Radiology,

Wycombe Hospital, High

Wycombe, Buckinghamshire, UK
\end{abstract}

\section{Correspondence to}

Dr Gwilym James Webb,

Department of Gastroenterology,

Wycombe Hospital, Queen

Alexandra Road, High Wycombe,

Buckinghamshire HP11 2TT, UK;

gwilym.webb@doctors.org.uk

Received 3 August 2013

Accepted 7 August 2013

Published Online First

3 September 2013
To cite: Webb GJ,

Chapman TP, Cadman PJ,

et al. Frontline

Gastroenterology 2014;5:

60-67.

\begin{abstract}
Pyogenic liver abscess has a variable clinical presentation. Its management requires input from several disciplines and is often coordinated by a gastroenterologist. This review examines demographics, clinical presentation, aetiology, diagnosis and prognosis; a suggested management approach, including antibiotic selection, radiological intervention and indications for surgery, is offered from a physician's perspective.
\end{abstract}

\section{INTRODUCTION}

Pyogenic liver abscess (PLA) is an uncommon condition but its incidence is increasing and it carries an appreciable mortality. ${ }^{12}$ Over recent years, there has been a trend away from surgery as initial management, with aggressive antibiotic treatment and percutaneous aspiration or drainage forming the mainstay of treatment. ${ }^{3}$ The aetiology of PLA has changed and iatrogenic interventional techniques, such as transarterial chemoembolisation and radiofrequency ablation of tumours, have been implicated. ${ }^{4}$ Furthermore, Klebsiella has recently emerged as a major causative organism, with a particularly high prevalence in the Far East. ${ }^{5}$

Recommendations for management of PLA are impaired by a lack of good-quality randomised trials, with most evidence arising from case series and retrospective reviews. In our experience, the care of patients with PLA is increasingly coordinated by gastroenterologists or hepatologists and we present this review with such physicians in mind. Familiarity with hepatic and gastrointestinal infections such as PLA is required in the UK specialty training curriculum for gastroenterology 2010.

\section{DEFINITION AND DEMOGRAPHICS}

PLA may be defined as an infectious space-occupying lesion in the liver with pyogenic bacteria as the causative agent. ${ }^{6}$ The incidence of PLA is rising: a large American series has described an incidence of 3.59 cases per 100000 population per year and this rate is increasing at $4 \%$ a year ${ }^{1}$; similar data are lacking for the UK. The same study described a male predominance with an OR of 1.85 and a correlation with increasing age: the group most likely to be affected being 65-84 years of age with an OR of 11.9 compared with those aged 18-34 years. In the US series, the comorbidities most commonly associated with PLA were $3.3 \%$ with prior liver transplantation, $16.2 \%$ with a previous diagnosis of any cancer and $21.9 \%$ with known biliary pathology.

\section{PRESENTATION}

The classic presentation of PLA is the triad of fever, right upper quadrant pain or fullness and jaundice, which is associated with pylephlebitis. ${ }^{2}$ However, this triad is relatively uncommon: a review of symptoms at presentation amongst a US cohort reported fever (89.6\%), right upper quadrant pain $(72.2 \%)$ and chills $(69.0 \%)$ as common; other symptoms included nausea, vomiting, weight loss, headache, myalgia and diarrhoea. Jaundice as a symptom was relatively uncommon and occurred in only $21.4 \% .^{7}$ Symptoms may be long-standing and may be mistaken for malignancy in more indolent cases.

The most commonly reported laboratory abnormalities from the study above ${ }^{7}$ included hypoalbuminaemia (70.2\%), leucocytosis (68.0\%), elevated alkaline phosphatase (66.7\%), elevated alanine aminotransferase $(53.8 \%)$ and hyperbilirubinaemia $(53.0 \%)$. C-reactive protein is often raised-in $100 \%$ of cases in one series. ${ }^{3}$ Initial blood cultures are positive 
in around half of patients. ${ }^{6}$ The mainstay of diagnosis of PLA is, however, imaging and this is described below.

\section{AETIOLOGY}

An assessment of the aetiology of PLA may be classified into causative organisms and causative associated diseases and procedures. Several large cases series have assessed the causative organism in cases of PLA. The most common bacterial isolates from blood or pus cultures among US patients are Streptococcus species, followed by Escherichia, Staphylococcus and Klebsiella. ${ }^{1}$ Klebsiella is the most common causative organism among patients from the Far East and it is also increasingly reported in nearby areas and migrant populations from the Far East. ${ }^{5}$ Rates of polymicrobial infection range from $11 \%$ to $65 \% .^{2}$

Klebsiella pneumoniae, particularly serotypes K1 and $\mathrm{K} 2$, has recently been identified as a cause of PLA associated with metastatic infection and is particularly common in people originating from Taiwan, China and the Far East. $K$ pneumoniae is asymptomatic in many of those from the Far East and has become the most common cause of PLA in that region. $K$ pneumoniae PLA appears to be associated with diabetes mellitus. Although the initial presentation is similar to PLA caused by other bacteria, distant infection, including high rates of bacteraemia, endophthalmitis, meningitis, septic pulmonary emboli and empyema, is reported. A finding of $K$ pneumoniae infection outside the liver should prompt examination for occult PLA. ${ }^{5}$ Other pathogens of note include Yersinia enterocolitica, which is reported to cause PLA in patients with haemochromatosis ${ }^{2}$ and Staphylococci, which are prevalent in cases associated with trauma. ${ }^{6}$ Microbial causes are summarised in box 1 .

The aetiology of PLA may be split into six categories: biliary infection, portal vein seeding (pylephlebitis), direct extension, hepatic arterial seeding, penetrating trauma and cryptogenic cause. ${ }^{8}$ The series

\section{Box 1 Major causative organisms}

- Streptococcus species (29.5\%; including enterococcus)

- Escherichia species (18.1\%)

- Staphylococcus species (10.5\%; NB associated with trauma)

- Klebsiella species $(9.2 \%$; NB higher rates in the Far East)

- Anaerobes (8.6\%; including Bacteroides species)

- Pseudomonas species $(3.1 \%)$

- Proteus species $(0.8 \%)$

- Remainder: rarer species or culture negative

NB Frequently polymicrobial; rates given are cases from where blood or abscess aspirate culture was positive in a large US series. Adapted from Meddings et al. ${ }^{1}$ from Johns Hopkins that first defined these categories described a shift from appendicitis with transvenous spread as the major cause of PLA to biliary occlusion, commonly by cholangiocarcinoma and especially after palliative stenting. ${ }^{8}$ The resultant cholangitis commonly causes multiple small PLAs. Rates vary, but the coexistence of malignancy is common and has been reported as ranging between $16.2 \%^{1}$ in a general hospital and $86 \%$ in a specialist surgical centre. ${ }^{4}$ An association with radiofrequency ablation and transarterial chemoembolisation has also been described and these procedures are associated with infections from skin commensals such as Streptococci and Staphylococci. ${ }^{4} 8$ Finally, recent work has suggested a link between PLA and subclinical colonic carcinoma. This association seems more marked among those with Klebsiella infection, although whether all patients with PLA should undergo colonoscopy remains uncertain. 59

Immunosuppression should also be considered in the aetiology of PLA. Potential causes include systemic chemotherapy, immunosuppressant drugs in transplant recipients and those with inflammatory and autoimmune disease, inherited immunodeficiency syndromes and acquired immunodeficiency states such as HIV/AIDS. ${ }^{6} 10$

Major aetiologies are summarised in box 2.

\section{DIAGNOSIS}

Imaging is the mainstay of diagnosis in PLA. Plain radiographs are insensitive and often normal but may show pleural effusion in around one-quarter and

\section{Box 2 Aetiologies in pyogenic liver abscess}

Biliary obstruction with cholangitis

- Malignant

- Biliary stenting

- Choledocholithiasis

- Malformation-for example, Caroli's disease

- Ascaris lumbricoides

Portal bacteraemia

- Diverticulitis

- Appendicitis

Direct invasion

- Cholecystitis

- Perinephric abscess

- Subphrenic abscess

Systemic bacteraemia

- Indwelling catheter infection

- Endocarditis

- Severe sepsis

Traumatic

- Direct trauma

- Radiofrequency ablation or tumour embolisation

- Sickle cell disease

Cryptogenic

- NB immunodeficiency 


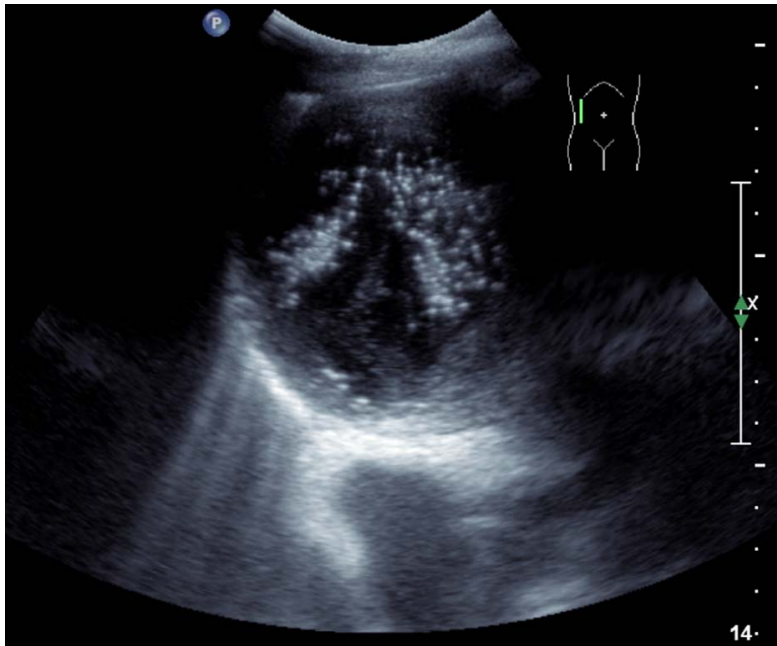

Figure 1 Abdominal ultrasound image of a pyogenic liver abscess. Note fluid-filled lesion with echogenic debris, septation and air bubbles.

extra-luminal free air on abdominal films in $7 \% .^{11}$ Ultrasonography is reported to have $94 \%$ sensitivity for PLA, allows assessment of the biliary tree and is widely available and therefore an appropriate initial imaging modality ${ }^{2}$ (figure 1). Ultrasonography may, however, not show the presence of smaller abscesses and is less able to distinguish metastases from abscesses than CT. MRI reliably demonstrates PLAs as $\mathrm{T}_{1}$ hypointense, $\mathrm{T}_{2}$ hyperintense lesions, but is not favoured for diagnosis because increased time is required for image acquisition, it is unsuitable for drainage guidance and has lower sensitivity in detecting other intra-abdominal pathology than CT. ${ }^{2} 67$

CT with intravenous contrast is the preferred imaging modality for PLA with a sensitivity approaching $100 \% .^{4} 711$ PLAs appear isodense with normal parenchyma on unenhanced CT, whereas they display relatively low attenuation after the administration of intravenous contrast. Typically, PLAs demonstrate rim-enhancement or a peripheral capsule. ${ }^{4}{ }^{6}$ As well as confirming the diagnosis, CT allows assessment of the presence and number of septations, the presence of multifocal abscesses and accurate sizing, which in turn guides decisions for drainage options. CT may show the presence of concurrent malignancy and potentially infective sources such as appendicitis, diverticulitis, cholecystitis (eg, figure 2) or biliary obstruction. ${ }^{16}$ Gas formation within PLAs is readily identified by CT and may indicate poorer prognosis. ${ }^{4}$ Furthermore, CT may demonstrate features such as a markedly thick oedematous abscess wall with few or no septations suggestive of the alternative diagnosis of amoebic abscess, ${ }^{5} 12$ which is less likely to require drainage and requires different antimicrobial treatment. ${ }^{2} 6$ The most common findings in PLA are of a single, large $(>5 \mathrm{~cm})$ non-enhancing lesion with a predilection for the right hepatic lobe (figure 3 ) but such



Figure 2 Transverse image from abdominal CT scan: small pyogenic liver abscess adjacent to the gallbladder. This case was a complication of acute calculous cholecystitis. Antibiotic monotherapy was successful and cholecystectomy was planned for later.

findings are variable (figures 4 and 5). ${ }^{5}{ }^{13}$ The differential diagnoses of amoebic abscess, echinococcusassociated abscess, tumour, tumour-associated PLA, simple cyst or biliary abnormality should be considered at the point of imaging, and further tests such as amoebic serology, echinococcus serology and tumour markers may be useful. ${ }^{2} 6$

Radiological diagnosis is supported by positive bacterial culture from either peripheral blood or abscess aspirate. $^{2}{ }^{6}$ However, over $30 \%$ of cases may be culture negative, with a lower yield if antibiotics are started before drawing cultures. ${ }^{78}$

\section{PROGNOSIS}

Untreated, PLA is almost uniformly fatal. ${ }^{2}$ There has, however, been a significant downward trend in mortality over the past 50 years. ${ }^{18}$ A recent large US case series



Figure 3 Transverse image from abdominal CT scan: large, multiseptate right lobe pyogenic liver abscess. 


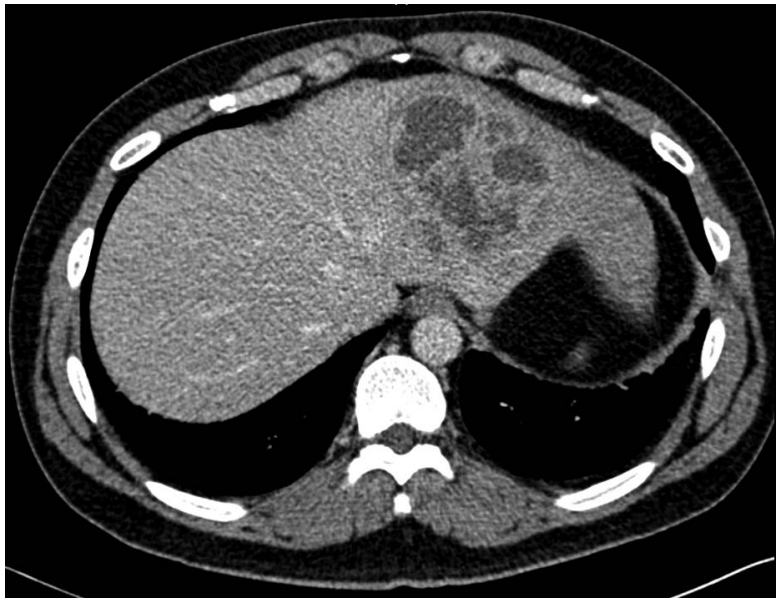

Figure 4 Transverse image from abdominal CT scan: multiseptated left lobe pyogenic liver abscess. The causative agent in this case was Klebsiella, although there are no features on CT that distinguish Klebsiella from other bacterial pathogens.

has reported in-hospital mortality of $5.6 \%,{ }^{14}$ a French series reported $8.7 \%^{13}$ and two British series $2.8 \%{ }^{10}$ and $11 \% .{ }^{14}$ Factors associated with a worse outcome in the US series included advanced age, bacteraemia, presentation with sepsis and concurrent diagnoses of renal failure, cirrhosis and cancer.

\section{MANAGEMENT}

\section{Antibiotics}

Antibiotic regimens used in suspected and confirmed PLA vary. General advice is that in suspected PLA, blood cultures are drawn before starting antibiotics, but treatment should not be delayed to allow aspiration unless this is imminent. ${ }^{2}$ There are no trials comparing antibiotics in PLA but common combinations used are summarised in box 3 . It is suggested that metronidazole is used in all suspected cases of PLA to cover anaerobic or amoebic infection ${ }^{2}$ :

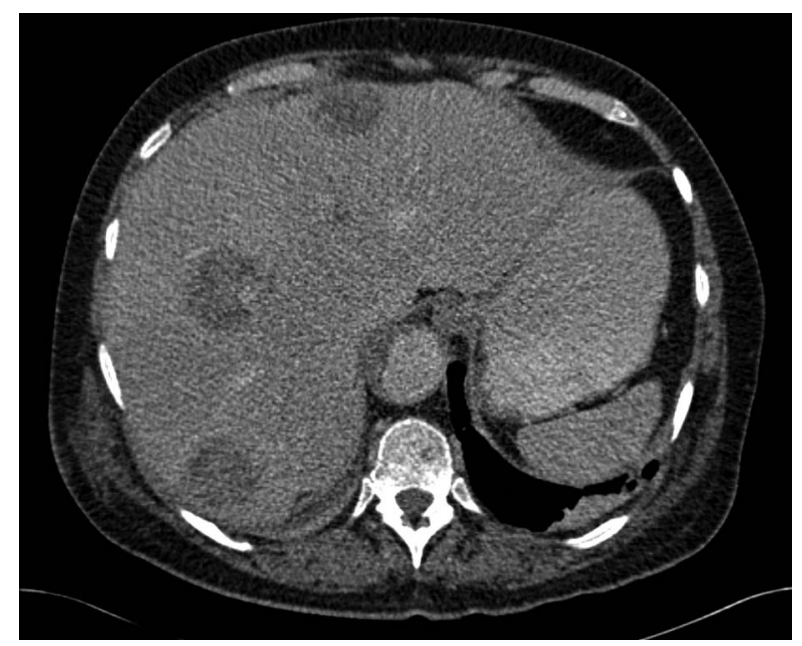

Figure 5 Transverse image from abdominal CT scan: three small separate pyogenic liver abscesses resulting from a diverticular abscess.

\section{Box 3 Potential antibiotic regimens}

- Third-generation cephalosporin (eg, ceftriaxone) and metronidazole

- Ampicillin, gentamicin and metronidazole

- Vancomycin, gentamicin and metronidazole

- Carbapenem (eg, imipenem or meropenem) and metronidazole

- Anti-pseudomonal penicillin with $\beta$-lactamase inhibitor (eg, piperacillin-tazobactam) and metronidazole

- Fluoroquinolone (eg, ciprofloxacin) and metronidazole \pm aminoglycoside

anaerobic organisms may be especially challenging to culture. Antibiotic regimens should be adjusted to culture results and isolate sensitivities. In suspected Klebsiella infections, third-generation cephalosporins with their superior central nervous system penetration are recommended while tight glycaemic control may reduce levels of bacteraemia. ${ }^{5}$

The optimal duration of antibiotic treatment is not clear, although a total duration of treatment of $<4$ weeks may be associated with a greater requirement for further intervention. ${ }^{12} \mathrm{~A}$ common treatment regimen for PLA is of a 6-8 week course of antibiotics. Typically, this consists of at least 2 weeks' intravenous treatment, with a conversion to oral agents when clinical and inflammatory responses allow. ${ }^{14}$ A recent Chinese study has suggested that stopping antibiotic treatment when C-reactive protein levels have normalised is a safe strategy, with a reported mean antibiotic duration of 16 days. ${ }^{15}$

All patients with PLA should receive antibiotics and, in some, antibiotic treatment alone is sufficient. A significant majority of single abscesses $\leq 3 \mathrm{~cm}$ may respond to antibiotic monotherapy without drainage. ${ }^{16}$ Treatment failure is more common with larger abscesses, reaching over $50 \%$ when monotherapy is attempted with abscesses up to $6 \mathrm{~cm}$ in diameter. ${ }^{3}$ For larger abscesses, some authors suggest drainage if there is a lack of clinical response to antibiotics, ${ }^{12}$ whereas others suggest drainage at diagnosis. ${ }^{16}$

\section{Percutaneous aspiration or drainage}

Successful percutaneous drainage of PLA was first reported in 1953 and has since become the mainstay of treatment for the condition. ${ }^{2}$ In comparison with surgical drainage, advantages of percutaneous drainage include its more rapid availability, the avoidance of general anaesthesia and smaller incision size.

Some interventional radiologists recommend simple aspiration as opposed to catheter drainage of PLA and in trials of smaller lesions they may be equivalent. Intervention is usually guided by ultrasound or CT. The data above suggest that antibiotic monotherapy is 
likely to be insufficient in abscesses $>3 \mathrm{~cm}$ or with loculations, and aspiration or drainage catheter placement is then appropriate. If a drainage catheter is placed, our opinion is that it should be irrigated three times a day with normal saline $(0.9 \%$ sodium chloride) and be removed when output is $<10 \mathrm{ml} /$ day. Potential complications of placement include causing communicating infection such as empyema and subphrenic abscess, haemorrhage and pneumothorax. Quoted success rates for percutaneous intervention for PLA range from $70 \%$ to $98 \%,{ }^{2} 6$ although presentation with gas-forming PLA or septic shock is associated with failure of percutaneous drainage and a subsequent requirement for surgery. ${ }^{13}$

\section{Biliary intervention}

Biliary infection is the most common cause of PLA and may cause multiple small abscesses. ${ }^{6}$ Where biliary infection is considered, dedicated biliary imaging should be carried out and any obstruction relieved by endoscopic retrograde cholangiopancreatography or percutaneous transhepatic cholangiography. ${ }^{10}$

\section{Indications for surgical referral}

In a modern series, over $90 \%$ of liver abscesses are treated without surgical intervention. ${ }^{1}$ However, surgery should be considered when very large, multiseptate or multifocal abscesses are present. This may be to permit surgical drainage or for hepatic resection. ${ }^{13}$



Figure 6 Suggested approach to the management of pyogenic liver abscess. Gl, gastrointestinal; PLA, pyogenic liver abscess. 
Multi-loculated PLAs $>5 \mathrm{~cm}$ in size may have a more favourable outcome with surgery than with percutaneous drainage. ${ }^{12}$ Overall, surgical intervention is associated with a worse outcome but this may represent selection bias of more severe cases. ${ }^{11}$ Underlying disease such as malignancy or biliary abnormality may require surgical resection; cases where percutaneous drainage has failed or is not technically feasible should also be considered for surgical drainage or resection. ${ }^{3}{ }^{16}$ In the rare complication of abscess rupture or fistulation, surgery is extremely likely to be required. ${ }^{11}$

\section{Investigating aetiology}

Other than explanatory abnormalities identified on initial CT, further focused testing may be useful for investigating the cause of PLA. Magnetic resonance cholangiography is valuable in identifying biliary disease, MRI of the liver may detect parenchymal abnormality or help to further characterise suspected tumour deposits, and colonic imaging or endoscopy may identify diverticular disease or tumour. Such studies, especially those of the biliary tree, are recommended if there is recurrent PLA. ${ }^{10}$ Although focused testing is the optimal approach to selecting further investigations depending on the aetiology, variations in clinical presentations may guide decision-making. For example, concurrent iron deficiency anaemia may suggest occult gastrointestinal blood loss from a tumour, whereas persistently deranged liver function tests may suggest a biliary abnormality. Equally, where there is a clear history of a route of infection, such as transarterial chemoembolisation or septicaemia from another source, further investigation may not be warranted.

\section{Follow-up}

Optimal follow-up is not clearly defined, but in addition to symptom resolution, normalisation of serum inflammatory markers should be achieved and serial imaging should confirm diminishing abscess size. Repeated abdominal CT scanning exposes patients to excess ionising radiation and serial ultrasound scanning can be used to ensure that the PLA size is reducing in response to antibiotics/drainage. Some recommend an interval CT to confirm resolution and look for residual, previously hidden malignancy. ${ }^{6}$

A suggested approach to the management of PLA is presented in figure 6 .

\section{CONCLUSION}

PLA is an uncommon infective condition that may present to a variety of services including gastroenterology, but requires input from microbiology, radiology, gastroenterology/hepatology and, in some cases, surgery. Diagnosis is primarily with appropriate imaging such as abdominal CT and culture of causative organisms where possible. Consideration should be given to invasive Klebsiella infection and to any associated causative condition (box 2), which might alter management or require separate treatment. Management is with appropriate antibiotic treatment, aspiration or drainage for all but the smallest abscesses, and surgery should be considered for resistant or complicated cases (figure 6, box 3). With such treatment mortality has decreased significantly, but is still appreciable.

\section{Multiple choice questions}

1. What is the preferred imaging modality for the diagnosis of pyogenic liver abscess (PLA)?

A. Computed tomography (CT) of abdomen

B. Erect abdominal radiography

C. Ultrasonography

D. MRI of the liver

E. Endoscopic retrograde cholangiopancreatography (ERCP)

Answer: [A]. CT provides the most sensitivity in the diagnosis of PLA, it also allows accurate sizing, planning for intervention and assessment of associated malignancy and other intra-abdominal pathology. It is widely available. Ultrasonography $[C]$ is appropriate but less sensitive than $\mathrm{CT}$, and $\mathrm{CT}$ would be recommended even after ultrasonographic diagnosis. MRI [D] may demonstrate PLA but there is less experience of its use, it is less available, may be less appropriate for sick patients and is less well suited to imaging the other intra-abdominal organs. ERCP may become necessary if imaging or intervention to the biliary tree is required [E] but will not reliably diagnose PLA. [B] Plain abdominal radiography is insensitive for PLA.

2. A patient with a past history of type II diabetes mellitus and hypertension presents with several days of fevers, malaise and jaundice. There is marked dyspnoea, meningism, photophobia and impaired vision with hypopyon in his left eye. The patient originates from China, although has not returned there for more than 1 year. Initial imaging suggests a multi-loculated liver abscess. Infection with which organism should be considered?

A. Streptococcus spp.

B. Staphylococcus spp.

C. Klebisella pneumoniae

D. Entamoeba histolytica

E. Escherichia coli

Answer: $[C]$. The combination of PLA with central nervous system infection (meningitis and endophthalmitis) should trigger consideration of Klebsiella infection. Diabetes and East Asian origin appear to be risk factors for Klebsiella infection. The other microorganisms listed are not commonly associated with central nervous system infection. 
3. A female patient presents with fever, deranged liver function tests, right-upper quadrant discomfort. She had lived in Egypt until 6 months previously. An abdominal CT scan is obtained (figure 7). Aspiration of the hepatic lesion is culture negative and there is no response to intravenous ceftriaxone. Which of the following diagnoses is most likely?

A. Echinococcosis

B. Liver metastasis

C. Biliary obstruction

D. Amoebiasis

E. Haemangioma

Answer: [D]. The CT scan demonstrates a large hypodense, fluid-filled abnormality in the right lobe of the liver. There are no septations and the wall is thick and oedematous. This is most consistent with an amoebic abscess. The travel history and lack of response to ceftriaxone together with negative culture support this diagnosis, which may be confirmed by serological studies. Liver abscesses secondary to echinococcus [A] are typically cystic and present less acutely. There is no evidence of biliary obstruction [C] or haemangioma [E]; liver metastases [B] are not typically fluid filled.

4. Which of the following bacteria appear to cause PLA with an increased frequency in patients with haemochromatosis?
A. A. Staphylococcus aureus
B. Klebsiella spp.
C. Escherichia spp.
D. Pseudomonas spp.
E. E. Yersinia enterocolitica

Answer: [E] Yersinia enterocolitica. All the listed bacteria are causes of PLA; however, Yersinia enterocolitica is a relatively rare cause except in those with haemochromatosis.

5. Which of the following presenting features is not a marker of poorer prognosis in patients with PLA?

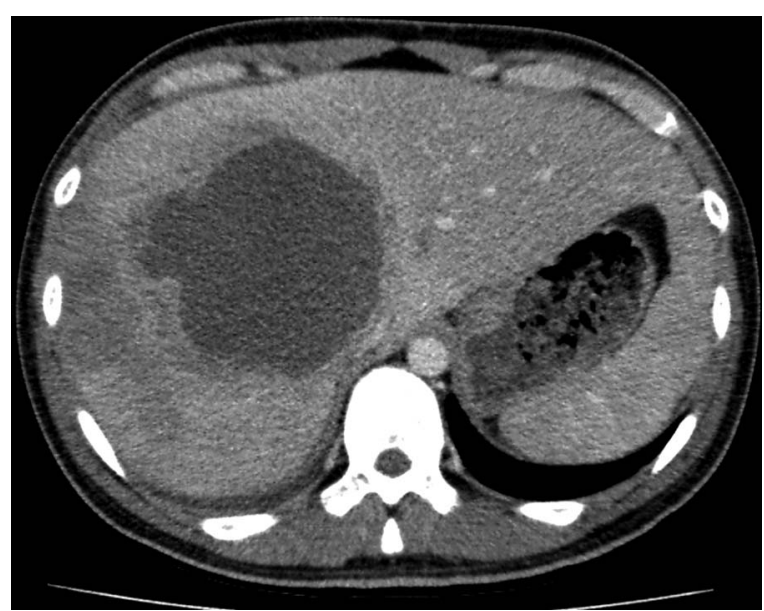

Figure 7 Multiple choice question 3.
A. Liver cirrhosis
B. Abscess $>5 \mathrm{~cm}$ in diameter
C. Renal failure
D. Requirement for percutaneous drainage
E. Septations within abscess

Answer: [D] Requirement for percutaneous drainage. All the other presenting features are associated with a worse outcome in a large case series; percutaneous drainage may be associated with an improved outcome.

6. Which of the following classes of antibiotic should be considered in all whenever treating suspected PLA?

A. Cephalosporin (eg, ceftriaxone)

B. Nitroimidazole (eg, metronidazole)

C. Aminoglycoside (eg, gentamicin)

D. Carbapenem (eg, meropenem)

E. Fluoroquinolone (eg, ciprofloxacin)

Answer: [B] Nitroimidazole (eg, metronidazole). All the antibiotics listed are potentially useful for the management of PLA. However, a nitroimidazole such as metronidazole is recommended in all cases to ensure coverage of anaerobic organisms, which may be challenging to culture. In addition, nitroimidazoles are the only listed antibiotic class with action against amoebic liver abscesses, which may be mistaken for PLAs.

Contributors GJW conceived the idea and drafted the manuscript. The other authors helped to revise the manuscript and contributed images.

Competing interests None.

Provenance and peer review Not commissioned; internally peer reviewed.

\section{REFERENCES}

1 Meddings L, Myers RP, Hubbard J, et al. A population-based study of pyogenic liver abscesses in the United States: incidence, mortality and temporal trends. Am J Gastroenterol 2009;105:117-24.

2 Johannsen EC, Sifri CD, Madoff LC. Pyogenic liver abscesses. Infect Dis Clin North Am 2000;14:547-63.

3 Heneghan HM, Healy NA, Martin ST, et al. Modern management of pyogenic hepatic abscess: a case series and review of the literature. BMC Research Notes 2011;4:80.

4 Mezhir JJ, Fong Y, Jacks LM, et al. Current management of pyogenic liver abscess: surgery is now second-line treatment. J Am Coll Surg 2010;210:975-83.

5 Siu LK, Yeh K-M, Lin J-C, et al. Klebsiella pneumoniae liver abscess: a new invasive syndrome. Lancet Infect Dis 2012;12:881-7.

6 Kurland JE, Brann OS. Pyogenic and amebic liver abscesses. Curr Gastroenterol Rep 2004;6:273-9.

7 Rahimian J, Wilson T, Oram V, et al. Pyogenic liver abscess: recent trends in etiology and mortality. Clinical infectious diseases 2004;39:1654-9.

8 Huang CJ, Pitt HA, Lipsett PA, et al. Pyogenic hepatic abscess. Changing trends over 42 years. Ann Surg 1996;223:600. 
9 Jeong SW, Jang JY, Lee TH, et al. Cryptogenic pyogenic liver abscess as the herald of colon cancer. J Gastroenterol Hepatol 2012;27:248-55

10 Pearce NW, Knight R, Irving $\mathrm{H}$, et al. Non-operative management of pyogenic liver abscess. HPB: Official Journal of The International Hepato Pancreato Biliary Association 2003;5:91-5.

11 Alvarez Pérez JA, Gonz'lez JJ, et al. Clinical course, treatment and multivariate analysis of risk factors for pyogenic liver abscess. Am J Surg 2001;181:177-86.

12 Chung YF, Tan YM, Lui HF, et al. Management of pyogenic liver abscesses-percutaneous or open drainage? Singapore Med J 2007;48:1158-65.
13 Alkofer B, Dufay C, Parienti JJ, et al. Are pyogenic liver abscesses still a surgical concern? A western experience. $H P B$ Surg 2012;2012:1-7.

14 Bosanko NC, Chauhan A, Brookes M, et al. Presentations of pyogenic liver abscess in one UK centre over a 15 -year period. JRCPE 2011;41:13-7.

15 Gao H-N. Clinical significance of C-reactive protein values in antibiotic treatment for pyogenic liver abscess. World J Gastroenterol 2010;16:4871.

16 Hope WW, Vrochides DV, Newcomb WL, et al. Optimal treatment of hepatic abscess. Am Surg 2008;74:178-82. 